\title{
Avaliação de parâmetros ecológicos da parasitofauna de duas espécies de peixes do Rio Mogi Guaçu, Região Sudeste do Brasil
}

\author{
Cintia Moreira Ramos dos Reis ${ }^{1}$, Náira da Silva Campos², Marlene Tiduko Ueta', Julio César José da \\ Silva², Paulo Sérgio Ceccarelli ${ }^{3} \&$ Silmara Marques Allegrette ${ }^{1}$
}

1'Instituto de Biologia (IB), Departmento de Biologia Animal, Programa de Pós Graduação em Biologia Animal, Laboratório de Helmintologia, Universidade Estadual de Campinas (UNICAMP), Campinas, SP, Brasil. Email: cinmoreira@gmail.com; mtu@unicamp.br; sallegre@unicamp.br.

${ }^{2}$ Instituto de Ciências Exatas (ICE), Departmento de Química Analitica, Programa de Pós Graduação em Química Analitica, Laboratório Baccan Universidade Federal de Juiz de Fora (UFJF), Juiz de Fora, MG, Brasil. Email: julio. silva@ufjf.edu.br; nairacampos@ice.ufjf.br.

${ }^{3}$ Instituto Chico Mendes de Conservação da Biodiversidade (ICMBio), Laboratório de Saúde, Bem Estar e Controle de Enfermidades, Pirassununga, SP, Brasil. Email: paulo.ceccarelli@icmbio.gov.br.

\begin{abstract}
Evaluation of ecological parameters of fauna prasite of two species of fish from the Mogi Guaçu River, southeast region of Brazil. The basin of the Moga Guaçu River has one of the best studied fish faunas in Brazil. Their fish are an important food source for the region's inhabitants. The objectives of this study were to analyze ecological aspects about the parasite fauna found in two fish species in this river. The objectives of this study were to survey and analyze the parasite fauna found in this river. We collected 229 specimens of Prochilodus lineatus, known as curimbatá, and 130 specimens of Pimelodus maculatus, known as yellow mandi, during the year 2012, in Cachoeira de Emas, municipality of Pirassununga, São Paulo. We analyzed the organs for collection of parasites and removed intestinal parasites for parasitological analysis. The total of 2,437 parasites were collected from $P$. lineatus, with the largest occurring in monogeneans gills $(n=1,425)$. Pimelodus maculatus presented a total of 4,742 parasites collected with parasite fauna similar to other rivers studied, with $99.2 \%$ of infected individuals for at least one species. Also, in this fish species, monogenean were the most prevalent parasites $(n=3,870$ ), which occurred in nearly all the individuals collected. The parasite fauna found were related to other already studied rivers.
\end{abstract}

Keywords: condition factor, helminths, Prochilodus lineatus, Pimelodus maculatus.

Resumo. A bacia do rio Mogi Guaçu tem uma das ictiofaunas mais bem estudadas do Brasil. Seus peixes representam importante fonte de alimento para os habitantes da região. Os objetivos deste trabalho foram analisar aspectos ecológicos da parasitofauna encontrada em duas espécies de peixes neste rio. Foram coletados 229 espécimes de Prochilodus lineatus, conhecido como curimbatá, e 130 espécimes de Pimelodus maculatus, conhecido como mandi-amarelo durante o ano de 2012, em Cachoeira de Emas, município de Pirassununga, São Paulo. Foram analisados os órgãos para coleta e retirada os parasitos intestinais para análise. $\mathrm{O}$ total 
de 2.437 de parasitos foi coletado de $P$. lineatus, sendo a maior ocorrência de monogenéticos em brânquias $(\mathrm{n}=1.425)$. Pimelodus maculatus apresentou um total de 4.742 parasitos coletados, com fauna parasitológica semelhante à de outros rios estudados, com 99,2\% dos indivíduos parasitados, por pelo menos uma espécie. Também nesta espécie de peixe os parasitos mais prevalentes foram os monogenéticos ( $n=3.870$ ), que ocorreram em quase todos os indivíduos coletados. A parasitofauna encontrada apresentou relação com de outros rios já estudados.

Palavra-chave: fator de condição, helmintos, Prochilodus lineatus, Pimelodus maculatus.

\section{INTRODUÇÃO}

O rio Mogi Guaçu faz parte da bacia que leva o mesmo nome e nasce na região da Serra da Mantiqueira no estado de Minas Gerais e deságua no rio Pardo, estado de São Paulo (Espíndola et al., 2003). Duas espécies de peixes comercializadas no país no estado são encontradas neste rio, o curimbatá, Prochilodus lineatus (Valenciennes, 1837) (Characiformes, Prochilodontidae) e o mandi-amarelo, Pimelodus maculatus, La Cépède, 1803 (Siluriformes, Pimelodidae).

Prochilodus lineatus apresenta hábito alimentar iliófago e é potamódromo [espécie que migra sempre em água doce, dentro de um rio (MESQUITA, 2011)]. Ocorrem em abundância e são nativas do rio objeto deste estudo. Os lares de alimentação se encontram na parte média do rio Grande e, quando se aproxima o período de migração para a ocorrência da reprodução, os peixes param de se alimentar e formam cardumes que se deslocam rio acima em direção aos lares de reprodução, localizados entre os municípios de Pirassununga e Salto de Pinhal, ocorrendo em abundância e sendo nativa do rio. (GoDoY, 1975).
Pimelodus maculatus tem alimentação diversificada, incluindo insetos aquáticos e terrestres, moluscos, anelídeos, peixes, rotíferos, copépodos, material vegetal e sedimento. São de clima subtropical, de ambiente bentopelágico, esta distribuídos na América do Sul, nas bacias dos rios La Plata, Paraguai, Paraná e São Francisco. São ovulíparos, com período de desova entre dezembro a fevereiro nas bacias do rio Paraná e do rio São Francisco (Mesquita, 2011).

Os parasitos de peixes do rio Mogi-Guaçu são bem estudados, sendo que muitas espécies dos grupos Monogenea, Acanthocephala, Nematoda, Cestoda, Crustacea já foram coletadas e identificadas. No rio Mogi Guaçu, os estudos ictioparasitários datam da década de 1920 (Travassos, Artigas e Pereira, 1928). Desde então pesquisas de caráter descritivo foram realizadas, bem como algumas descrições de táxons parasitários vêm sendo publicadas (KoHn et al., 1985; KoHn et al., 1987 AgUIAR et al. 2011, MESQUitA, 2011; CEPEDA et al., 2012).

O presente estudo tem como objetivo estudar aspectos ecológicos relativos a fauna parasitária de $P$. lineatus e $P$. maculatus no rio 
Mogi-Guaçu ao longo do ano de 2012, bem como avaliar fatores de condição.

\section{Material e Métodos}

Os peixes foram capturados no rio Mogi Guaçu, no distrito de Cachoeira de Emas, município de Pirassununga (SP). O rio nasce no morro do Curvado, município de Bom Repouso, na região da Serra da Mantiqueira, estado de Minas Gerais a uma altitude de aproximadamente 1.650 m.s.m nas coordenadas $22^{\circ} 30^{\prime} \mathrm{S}$ e $46^{\circ} 08^{\prime}$ W e percorre $530 \mathrm{~km}$ desaguando no rio Pardo a 483 m.s.m. Faz parte da bacia do rio Mogi Guaçu que abrange uma área de $17.460 \mathrm{~km}^{2}$, sendo $2.650 \mathrm{~km}^{2}$ no estado de Minas Gerais e o restante no estado de São Paulo. Com uma extensão de $473 \mathrm{~km}$, distribui-se por 53 cidades (sendo 12 em Minas Gerais e 41 em São Paulo) (EsPíndola et al., 2003). O local de análise dos espécimes coletados foi o laboratório de Saúde, Bem-Estar e Controle de Enfermidades do CEPTA/ICMBio (Centro Nacional de Pesquisa e Conservação da Biodiversidade Aquática Continental / Instituto Chico Mendes de Conservação da Biodiversidade).

O trabalho foi aprovado pelo Comitê de Ética no Uso de Animais da Universidade Estadual de Campinas - CEUA/UnICAMP, protocolo número 2645-1, estando de acordo com os princípios éticos na experimentação animal adotados pela Sociedade Brasileira de Ciências em Animais de Laboratório (SBCAL) e com a legislação vigente, Lei №. 11.794, de oito de outubro de 2008 e o decreto №. 6.899, de 15 de julho de 2009.

Expedições mensais de coleta foram realizadas entre janeiro e dezembro de 2012, cobrindo os períodos de seca (março a agosto) e cheia (setembro a fevereiro) do rio, nas proximidades da barragem da Usina Hidrelétrica de Cachoeira de Emas, Pirassununga, SP $\left(21^{\circ} 55^{\prime}\right.$ S e $47^{\circ} 22^{\prime}$ W).

Foram coletados 229 espécimes de $P$. lineatus e 130 de $P$. maculatus com o auxílio de redes e tarrafas. Os peixes foram transportados vivos até o laboratório de Saúde, Bem-Estar e Controle de Enfermidades do CEPTA/ICMBio, onde foram eutanasiados mediante a transecção da coluna vertebral em curimbatá e comoção cerebral em mandi-amarelo.

Os peixes coletados foram identificados em laboratório, sendo cada espécime pesado e medido o comprimento (medida padrão). As brânquias de cada peixe foram colocadas em recipientes contendo 1:1 de água aquecida a 65 ㄷ $\mathrm{C}$ e formol $5 \%$ e a amostra foi agitada para desprendimento dos ectoparasitos, sendo o líquido analisado e os Monogenoidea coletados. Para análise das narinas foi feito um corte longitudinal em cada abertura das cavidades nasais e em seguida com auxílio de uma seringa foi feita a lavagem com 1:1 de água a $65^{\circ} \mathrm{C}$ e formol $5 \%$ e o líquido coletado para avaliação de ectoparasitos, esta metodologia foi adaptada de KRITSKY et al. (2007). Após a coleta das brânquias e lavagem das narinas deu-se a dissecção do animal; na região dorsal foi feito um corte longitudinal da parte posterior até a anterior do peixe. Bexiga natatória, esôfago, estômago e intestino foram coletados e colocados em placas 
de Petri contendo solução salina, e em seguida levados ao microscópio estereoscópico para a coleta de parasitos. Os helmintos encontrados foram fixados de acordo com AMATo et al. (1991).

Os peixes foram separados de acordo com os estádios gonadais, baseado em VAzzoLER (1981) e também por estação (seca e cheia). As médias de pesos e comprimentos foram utilizadas nos cálculos. As variáveis utilizadas foram mês de coleta, sexo e estádio gonadal, relacionados ao comprimento, peso e frequência parasitária, utilizando Análise de variância (ANOVA - One Way) com teste de média de Duncan. Os valores de relação peso-comprimento foram obtidos com base nas duas estações do ano (cheia e seca). Foi usado teste de Kruskal Wallis para análise de significância entre peso-comprimento para duas estações do ano, seca (março a agosto) e cheia (setembro a fevereiro). A prevalência, intensidade média e abundância média dos parasitos foram calculadas de acordo com BUSH et al. (1997).

\section{RESULTADOS}

\section{Prochilodus lineatus}

Foram coletados 229 P. lineatus 0 comprimento e o peso médios foram, $28,4 \pm 4,5$ cm e $514,1 \pm 260,0 \mathrm{~g}$. As fêmeas apresentaram média de peso de $480,5 \pm 228,1$ g e comprimento de $32,4 \pm 4,8 \mathrm{~cm}$. Os machos média de peso de $546,8 \pm 285,0$ g e comprimento médio de $33,7 \pm 5,9$ cm. Dentre os espécimes, 113 eram fêmeas, 66 imaturas (I), 29 em maturação (II), 12 maduras (III), seis fêmeas esgotadas e 116 machos, dos quais 65 jovens (I), 37 em maturação (II), 13 maduros e um esgotado.

As diferenças estatísticas em relação ao comprimento e os meses de coleta foram significativos ( $F=18,17$ e $p<0,0001$ ) e os meses em relação ao peso dos indivíduos também foram estatisticamente diferentes ( $F=18,13$ e $p<0,0001$ ) (Tabela 1). Os machos quando comparados com as fêmeas, apresentaram maiores médias de comprimento total $(33,7 \pm$ $5,9 \mathrm{~cm}$ e $32,4 \pm 4,8 \mathrm{~cm})$ e peso $(546,8 \pm 285,0 \mathrm{~g}$ e $480,5 \pm 228,1 \mathrm{~g})$, sendo as significativamente diferentes (comprimento, $F=148,9$ e $p<0,0001$; peso $\mathrm{F}=238,7$ e $\mathrm{p}<0,0001$ ).

Na estação de cheia as médias de peso e comprimento foram respectivamente $535,5 \pm$ 240,4 g e 33,9 $\pm 5,1 \mathrm{~cm}$ e, na estação de seca, o peso apresentou a média de 488,4 $\pm 280,8 \mathrm{~g}$ e o comprimento foi de $32,0 \pm 5,6 \mathrm{~cm}$. Já para a relação entre peso e estádio gonadal houve diferença estatística significativa ( $F=374,6$ e $p<$ $0,0001)$ e entre comprimento e estádio gonadal também apresentou diferença estatística significativa ( $F=404,4$ e $p<0,0001$ ) (Tabela 2).

O número de jovens foi maior ao longo de todo o ano. Houve diferença estatística significativa em relação aos meses de coleta e os estádios gonadais (Figura 1). Foi encontrado o total de 2.437 parasitos, sendo que destes, 1,425 foram monogenéticos de brânquias (Tabela 3), na narina foram encontrados 341 monogenéticos e no intestino 12 Digenea, todos pertencentes ao filo Platyhelminthes. O filo Nematoda apresentou 
Tabela 1. Médias de comprimento $(\mathrm{cm})$ e peso $(\mathrm{g})$ em relação aos meses de coleta Prochilodus lineatus no rio Mogi Guaçu, Cachoeira de Emas entre janeiro e dezembro de 2012.

\begin{tabular}{cccccccccccccccccccccccc}
\hline Mês & Jan & Fev & Mar & Abr & Mai & Jul & Ago & Set & Out & Nov & Dez \\
\hline Comprimento & $30,4^{\mathrm{B}}$ & $27,3^{\mathrm{E}}$ & $25,7^{\mathrm{G}}$ & $26,0^{\mathrm{G}}$ & $24,0^{\mathrm{H}}$ & $29,8^{\mathrm{C}}$ & $32,6^{\mathrm{A}}$ & $30,5^{\mathrm{B}}$ & $28,4^{\mathrm{D}}$ & $28,0^{\mathrm{D}}$ & $26,7^{\mathrm{F}}$ \\
Peso & $719,7^{\mathrm{B}}$ & $469,4^{\mathrm{E}}$ & $384,8^{\mathrm{F}}$ & $388,6^{\mathrm{F}}$ & $280,2^{\mathrm{G}}$ & $546,6^{\mathrm{C}}$ & $772,5^{\mathrm{A}}$ & $559,2^{\mathrm{C}}$ & $449,4^{\mathrm{E}}$ & $510,1^{\mathrm{D}}$ & $393,1^{\mathrm{F}}$ \\
\end{tabular}

Médias seguidas de letras iguais na mesma linha não diferem significativamente ao nível de $5 \%$ teste de médias de Duncan (ANOVA).

Tabela 2. Médias de comprimento $(\mathrm{cm})$ e peso $(\mathrm{g})$ para ambos os sexos de Prochilodus lineatus em relação ao estádio gonadal (I, II, III, IV) no rio Mogi Guaçu, Cachoeira de Emas, entre janeiro e dezembro de 2012.

\begin{tabular}{|c|c|c|c|c|c|c|c|c|c|c|c|}
\hline Mês & Jan & Fev & Mar & Abr & Mai & Jul & Ago & Set & Out & Nov & Dez \\
\hline Comprimento & $30,4^{\mathrm{B}}$ & $27,3^{\mathrm{E}}$ & $25,7^{G}$ & $26,0^{G}$ & $24,0^{\mathrm{H}}$ & $29,8^{c}$ & $32,6^{A}$ & $30,5^{B}$ & $28,4^{\mathrm{D}}$ & $28,0^{\mathrm{D}}$ & $26,7^{F}$ \\
\hline Peso & $719,7^{\mathrm{B}}$ & $469,4^{E}$ & $384,8^{F}$ & $388,6^{\mathrm{F}}$ & $280,2^{\mathrm{G}}$ & $546,6^{c}$ & $772,5^{A}$ & $559,2^{c}$ & $449,4^{\mathrm{E}}$ & $510,1^{D}$ & $393,1^{\mathrm{F}}$ \\
\hline
\end{tabular}

Médias seguidas de letras (maiúsculas) iguais na mesma linha não diferem significativamente ao nível de 5\% teste de médias de Duncan (ANOVA). I - jovens; II - em maturação; III - maduros e IV - esgotados

\section{Estádios gonadais em machos e fêmeas de $P$. lineatus por mês}

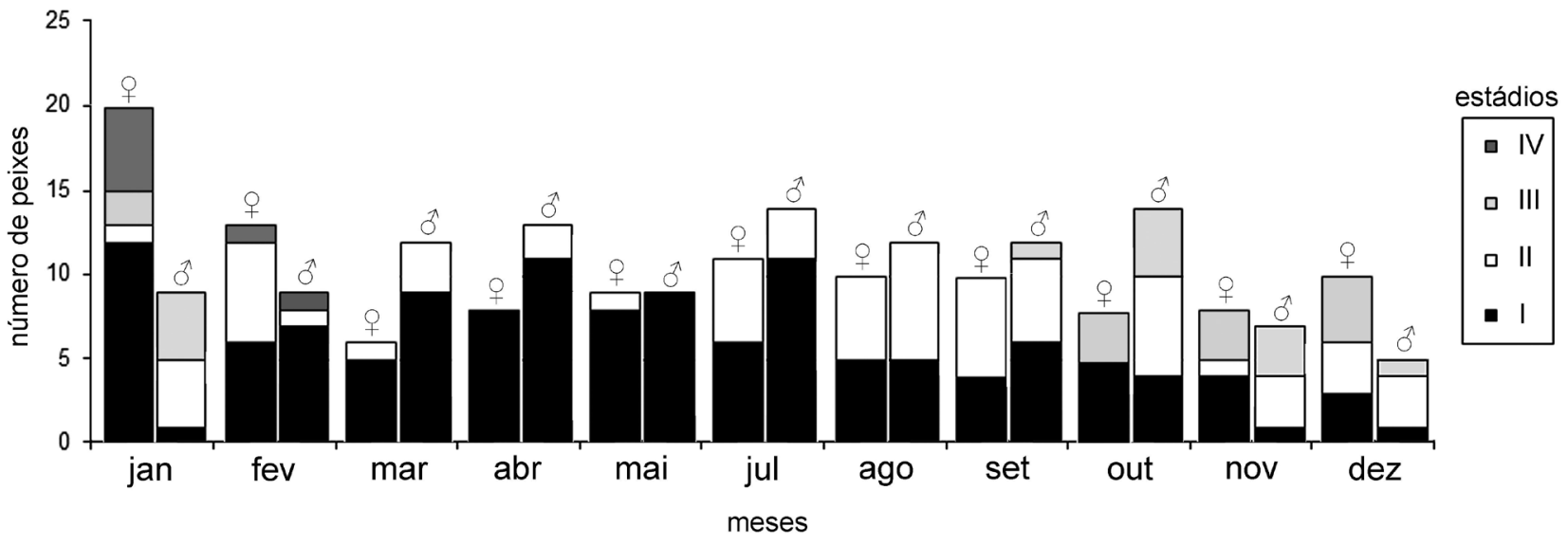

Figura 1. Relação dos estádios gonadais de machos e fêmeas (I, II, III, IV) para cada mês de coleta para Prochilodus lineatus no rio Mogi Guaçu, Cachoeira de Emas, entre janeiro e dezembro de 2012. 
Tabela 3. Número de espécimes registrados em 229 peixes e índices parasitológicos dos parasitos de Prochilodus lineatus, no rio Mogi Guaçu, Cachoeira de Emas, SP, Brasil, em 2012.

\begin{tabular}{|c|c|c|c|c|c|c|}
\hline Localização & Parasitos & № H & № $P$ & $\mathrm{P} \%$ & IM & AM \\
\hline \multirow{3}{*}{ Intestino } & Procamallanus sp BAYLIS, 1923 & 4 & 7 & 1,75 & 1,75 & 0.03 \\
\hline & $\begin{array}{c}\text { Neoechinorhynchus curemai } \\
\text { NORONHA, } 1973\end{array}$ & 17 & 61 & 7,42 & 3,59 & 0,27 \\
\hline & Saccocoeloides nanii SZIDAT, 1954 & 1 & 12 & 0,44 & 12,00 & 0,05 \\
\hline Esôfago & $\begin{array}{l}\text { Spinitectus asperus Travassos, } \\
\text { ARTIGAS \& PEREIRA, } 1928\end{array}$ & 11 & 27 & 4,80 & 2,45 & 0,12 \\
\hline Bexiga natatória & Klossinemella sp. CostA, 1961 & 39 & 217 & 17,03 & 5,56 & 0,95 \\
\hline Narina & $\begin{array}{l}\text { Rhinonastes pseudocapsaloideum } \\
\text { KRITSKY, THATCHER \& BOEGER, } 1988\end{array}$ & 127 & 326 & 55.46 & 2.57 & 1.42 \\
\hline \multirow{2}{*}{ Brânquia } & Larva gloquidio Bivalve & 10 & 17 & 4.37 & 1,70 & 0.07 \\
\hline & Monogenéticos & 164 & 1425 & 71,62 & 8,69 & 6,22 \\
\hline \multirow{2}{*}{$\begin{array}{l}\text { Brânquia, narina e } \\
\text { intestino }\end{array}$} & Placobdella maculata WEBER, 1915 & 76 & 279 & 33,19 & 3,67 & 0,34 \\
\hline & Myracetyma sp. MALTA, 1993 & 50 & 16 & 21,83 & 0,32 & 0,07 \\
\hline
\end{tabular}

№ H: número de hospedeiros infectados, № P: número de parasitos, P\%: prevalência, IM: intensidade média, AM: abundância média

espécies na bexiga, em um total de 217, no intestino foram coletados sete, no estômago três e no esôfago também três espécimes. No Filo Acanthocephala foram coletados 61 espécimes no intestino. Arthropoda na narina seis indivíduos e nas brânquias 44 indivíduos, Annelida foram coletados 35 indivíduos na narina, 235 nas brânquias, dois na bexiga natatória, quatro no intestino, dois no estômago e um no esôfago e Molusca foram coletados 18 espécimes nas brânquias.

Após esta análise foi calculado o $\mathrm{K}$ e realizado o teste de Kruskal-Wallis com a finalidade de verificar a significância dos valores encontrados. Os resultados mostraram que houve diferença estatística significativa para este parâmetro $(p<0,05$ e H $=164$,2) entre as estações de cheia e seca. $\mathrm{O}$ resultado obtido foi de $p<0,00002$. $O$ coeficiente $b$ para a estação de cheia foi 2,4527 com $R^{2}=0,2855$ e para a estação seca $b$ foi 3,1143 com $R^{2}=0,9382$.

\section{Pimelodus maculatus}

Foram coletados 130 P. maculatus sendo o comprimento médio total de $18,5 \pm 5,3$ $\mathrm{cm}$ e a média do peso $136,8 \pm 131,2 \mathrm{~g}$. A média de comprimento para as fêmeas foi de $22,9 \pm 6,9$ $\mathrm{cm}$ e para os machos foi de $21,0 \pm 3,8 \mathrm{~cm}$. Dentre 
estes, 78 eram fêmeas, sendo 45 imaturas (I), 25 maduras (II), oito maduras (III) e nenhuma fêmea esgotada. Dentre os machos foram coletados 36 imaturos (I), 16 em maturação (II), não havendo indivíduos de outros estádios. Na estação de cheia as médias de comprimento padrão e peso foram, respectivamente, $25,0 \pm 5,5 \mathrm{~cm}$ e $188,8 \pm 142,9 \mathrm{~g}$, na estação seca as médias encontradas foram para comprimento $18 \pm 3,5 \mathrm{~cm}$ e peso $56,1 \pm 52,8$ g.

Houve diferença significativa para os parâmetros comprimento ( $F=31,9$ e $p<0,0001)$ e peso $(F=38,9$ e $p<0,0001)$ entre os meses de coleta. Para as variáveis comprimento e peso comparadas aos meses de coleta, setembro foi o mês com maior comprimento $(31,1 \mathrm{~cm})$ e março o menor $(14,0 \mathrm{~cm})$ e os maiores pesos no mês de setembro $(560,7 \mathrm{~g})$ e os menores nos meses de maio (51,9 g), março $(46,2 \mathrm{~g})$ e abril $(45,8 \mathrm{~g})$ como mostrado na tabela a seguir para $P$. maculatus (Tabela 4). para as duas estações do ano, mostraram um crescimento alométrico negativo para o período de seca, onde $b=2,9621$ com $R^{2}=0,9308$ e alométrico positivo para o período de cheia com $b=3,4001$ e $\mathrm{R}^{2}=0,9448$. Não foram coletados em nenhum período do ano estádios esgotados. Houve diferença estatística para os parâmetros avaliados, sendo que para o peso em relação ao estádio gonadal foi encontrado $(F=155,6$ e $p$ $<0,0001$ ) e para o comprimento em relação ao estádio gonadal ( $F=143,9$ e $p<0,0001$ ) (Tabela 5).

A presença de indivíduos jovens ao longo do ano de coleta está demonstrada na Figura 2, sendo que para os meses de setembro e novembro não foram encontrados jovens machos para a espécie estudada e no mês de julho não foi encontrado nenhum indivíduo. Já os valores de prevalência, intensidade média e abundância média estão expressos em tabela (Tabela 6). Os monogenoides foram os mais prevalentes,

Tabela 4. Médias de comprimento $(\mathrm{cm})$ e peso $(\mathrm{g})$ em relação aos meses de coleta para Pimelodus maculatus no rio Mogi Guaçu, Cachoeira de Emas, SP, Brasil, entre janeiro e dezembro 2012.

\begin{tabular}{cccccccccccccccccccc}
\hline Mês & Jan & Fev & Mar & Abr & Mai & Ago & Set & Out & Nov & Dez \\
\hline Comprimento & $17,52^{\mathrm{D}}$ & $16,41^{\mathrm{E}}$ & $14,0^{\mathrm{G}}$ & $14,9^{\mathrm{F}}$ & $14,5^{\mathrm{F}, \mathrm{G}}$ & $19,9^{\mathrm{C}}$ & $31,07^{\mathrm{A}}$ & $25,07^{\mathrm{B}}$ & $25,73^{\mathrm{B}}$ & $19,24^{\mathrm{C}}$ \\
Peso & $87,15^{\mathrm{D}}$ & $98,0^{\mathrm{D}}$ & $46,17^{\mathrm{E}}$ & $45,8^{\mathrm{E}}$ & $51,9^{\mathrm{E}}$ & $151,0^{\mathrm{C}}$ & $560,6^{\mathrm{A}}$ & $298,9^{\mathrm{B}}$ & $308,0^{\mathrm{B}}$ & $103,4^{\mathrm{D}}$ \\
\hline
\end{tabular}

Médias seguidas de letras (maiúsculas) iguais na mesma linha não diferem significativamente ao nível de $5 \%$ teste de médias de Duncan (ANOVA).

Os valores encontrados para $P$. com 128 hospedeiros infestados (98\%), em um maculatus para a relação peso-comprimento, total de 3.807 monogenéticos coletados nas 
Tabela 5. Valores das médias de comprimento $(\mathrm{cm})$ e peso $(\mathrm{g})$ de Pimelodus maculatus em relação ao estádio gonadal (I, II, III, IV) no rio Mogi Guaçu, Cachoeira de Emas entre janeiro e dezembro de 2012.

\begin{tabular}{cccc}
\hline Estádio gonadal & I & II & III \\
\hline Comprimento & $16,2^{\mathrm{C}}$ & $21,3^{\mathrm{B}}$ & $26,5^{\mathrm{A}}$ \\
Peso & $81,0^{\mathrm{C}}$ & $200,9^{\mathrm{B}}$ & $364,9^{\mathrm{A}}$ \\
\hline
\end{tabular}

Médias seguidas de letras (maiúsculas) iguais na mesma linha não diferem significativamente ao nível de 5\% teste de médias de Duncan (ANOVA). Estádios gonadais: I - jovem; II - em maturação; III - maduro; IV - esgotado.

Tabela 6. Valores ecológicos de parasitos de 130 Pimelodus maculatus, capturados no rio Mogi Guaçu, Cachoeira de Emas, SP, Brasil, em 2012.

\begin{tabular}{|c|c|c|c|c|c|c|}
\hline Localização & Parasitos & № $\mathrm{H}$ & № $P$ & $\mathrm{P} \%$ & IM & AM \\
\hline \multirow{3}{*}{ Intestino } & $\begin{array}{c}\text { Procamallanus (Spirocamallanus) } \\
\text { pimelodus Magalhães Pinto, } \\
\text { Fabio, Noronha \& Rolas, } 1975\end{array}$ & 36 & 56 & 27,6 & 1,55 & 0,43 \\
\hline & $\begin{array}{c}\text { Cucullanus pinnai pinnai } \\
\text { Travassos, Artigas \& Pereira, } 1928\end{array}$ & 12 & 16 & 9,23 & 1,33 & 0,12 \\
\hline & Cestoda & 5 & 6 & 3,85 & 1,20 & 0,05 \\
\hline Bexiga natatória & Nematoda - larvas & 34 & 93 & 26,15 & 2,74 & 0,72 \\
\hline Narina & Monogenoidea & 108 & 631 & 83,08 & 5,84 & 4,85 \\
\hline Brânquia & Monogenoidea & 128 & 3870 & 98,46 & 30,23 & 29,77 \\
\hline $\begin{array}{l}\text { Brânquia e } \\
\text { Narina }\end{array}$ & Myracetyma sp. Malta, 1993 & 3 & 5 & 2,31 & 1,67 & 0,04 \\
\hline $\begin{array}{l}\text { Brânquia, narina } \\
\text { e bexiga } \\
\text { natatória }\end{array}$ & $\begin{array}{c}\text { Placobdella maculata Weber, } \\
1915\end{array}$ & 18 & 57 & 13,85 & 3,17 & 0,14 \\
\hline Musculatura & Nematoda larvas & 1 & 8 & 0,77 & 8,00 & 0,06 \\
\hline
\end{tabular}

№ H: número de hospedeiros infectados, № P: número de parasitos, P\%: Prevalência, IM: Intensidade média e AM: Abundância média. 
brânquias. Além das brânquias foram coletados nas narinas 631 monogenéticos. Foram coletados também do filo Platyhelminthes seis Cestodas. Do Filo Nematoda foram coletados 93 na bexiga natatória, 84 no intestino e oito na musculatura de um único individuo. Nos Annelidas 13 hirudineas na narina, 42 nas brânquias e dois na bexiga natatória. No Filo Arthropoda foram coletados quatro copepodos nas narinas e um nas brânquias Foram coletados um total de 4691 parasitos ao longo do estudo.

O coeficiente angular $b$ para a estação de cheia foi de 3,4001 e para a estação seca foi 2,9621. O teste de Kruskal Wallis resultou $p<$ 0,0001 e $\mathrm{H}=75,58$ e o valor encontrado para $\mathrm{K}$ foi $p<0,0003$, mostrando que houve diferença entre as estações do ano para a relação pesocomprimento para esta espécie.

\section{DIscussão}

O menor comprimento e peso das fêmeas em relação aos machos de $P$. lineatus é um resultado diferente dos valores encontrados por Mesquita (2011) no rio Mogi Guaçu. No entanto, apenas cinco coletas foram realizadas pelo autor, entre 2008 e 2010. Por outro lado, LIZAMA et al. (2005) encontraram para a mesma espécie de peixe na planície inundada do alto rio Paraná, valores mais próximos aos do presente trabalho, com peso médio de 566,3 g e comprimento médio de $25,6 \mathrm{~cm}$. Para a mesma espécie, no rio Miranda, alto rio Paraguai. VICENTIN et al. (2012) encontraram os comprimentos variando entre 17,0 a $52,9 \mathrm{~cm}$ para machos e 23,0 a 51,0 para fêmeas e o peso foi de 55,0 a $1.900 \mathrm{~g}$ para os machos e 150 a 2.000 g para fêmeas, para 772 indivíduos coletados (499 machos e 273 fêmeas), valores também próximos aos encontrados neste estudo no rio Mogi Guaçu.

BARBIERI et al. (2000b) no rio Mogi Guaçu também encontraram valores maiores de peso e comprimento, respectivamente, para machos e fêmeas $(561,4 \mathrm{~g}$ e $29,9 \mathrm{~cm}$, e $880,4 \mathrm{~g}$ e 35,6 cm). Estes autores relataram que fêmeas com maior taxa de crescimento, e em maior quantidade representam uma tática de reposição populacional, concluindo, ainda, que os peixes começaram a se reproduzir com precocidade. Também observaram que a redução da primeira maturação gonadal, poderia estar relacionada a uma tática adotada pela espécie, no sentido de recuperar o equilíbrio populacional. Observouse que os tamanhos mínimos dos exemplares adultos capturados foram menores que em anos anteriores, de modo que essa mudança ocorrida na população pode ter relação com fatores abióticos e bióticos (BARBIERI et al., 2004).

O número de $P$. lineatus adultos foi menor que de jovens (98 adultos versus 131 jovens). Mesmo para os meses de migração, correspondendo aos meses de setembro até fevereiro, a ocorrência de indivíduos adultos foi reduzida. Uma vez que este é o período em que os espécimes se deslocam para reproduzir, era esperada maior ocorrência de adultos do que de jovens. Indivíduos esgotados também foram pouco coletados, mas estes indivíduos foram encontrados apenas nos meses de reprodução, 
corroborando estudos de Godor (1975) e BARBIERI et al. (2000a). A presença de mais indivíduos jovens pode estar relacionada ao fato de existirem populações fixas desta espécie no local (BARBIERI et al. 2000b).

O local de coleta deste estudo foi considerado como sendo lar de reprodução, de modo que era esperado que, para os meses considerados de reprodução, houvesse maior ocorrência de indivíduos maduros ou esvaziados, fato não observado. BARBIERI (2000b) também relatou que a redução do comprimento dos indivíduos de uma população pode estar relacionada a ambientes impactados causando modificações fisiológicas e, caso essas alterações continuem, a espécie pode ser extinta localmente (Godoy, 1975; CAPELETI \& PETRERE JR., 2006;).

Para as espécies de parasitos coletados, a análise estatística mostrou que não houve diferença significativa entre os valores biométricos e a frequência parasitária. Assim, pode-se inferir que os parasitos não interferiram no desenvolvimento dos peixes, o que pode estar relacionado à baixa prevalência e abundância de parasitos coletadas, ou a maior presença de jovens ao longo do ano, pois é possível que peixes mais velhos apresentem maior número de parasitos. Assim no presente trabalho, como foi observado maior número de peixes em estádios iniciais, é possível que seja uma resposta para a baixa prevalência e abundância de parasitos, bem como a densidade populacional distinta de peixes pode ser um determinante na riqueza parasitária, bem como a presença ou não do hospedeiro intermediário pode influenciar nesta baixa prevalência (ТАKEMOTO et al., 2009).

Os valores ecológicos para os parasitos de $P$. lineatus no presente estudo podem ser considerados baixos. No entanto, quando comparados a outros trabalhos na mesma localidade, observa-se que tais valores também foram baixos, conforme apresentado por КонN et al. (1985; 1987) e MEsquitA (2011). O último autor, em trabalho realizado no mesmo ponto de coleta e com a mesma espécie de peixe, encontraram apenas três espécies de parasitos, sendo dois nematoides e um digenético. Dentre os 45 hospedeiros analisados apenas 12 (26,7\%) estavam infectados por pelo menos um parasito. O digenético (Saccocoeloides nanii, Szidat, 1954) e o nematóide Spinitectus asperus Travassos, Artigas \& Pereira, 1928, foram comuns ao presente trabalho. Apesar disso, os monogenoides apresentaram alta prevalência, fato que pode ser explicado pelo ciclo reprodutivo direto destes organismos. KoHN et al. (1985) encontraram para coletas realizadas no rio Mogi Guaçu uma prevalência de $60 \%$ de monogenóides.

Durante as duas estações do ano observou-se que para $P$. lineatus a relação pesocomprimento foi negativa na estação cheia e positiva na estação seca, ou seja, no período de seca (outono-inverno) houve maior investimento em peso do que em comprimento, e, para o período cheia (primavera-verão), este incremento em peso foi menor comparado ao comprimento 
destes animais. A cheia é a época em que ocorre a migração desta espécie, e o investimento em peso ocorre apenas para os indivíduos jovens e adultos em maturação que estão ainda se desenvolvendo e para os demais adultos o comprimento total já foi atingido. A seca é a fase em que os peixes se alimentam, entre março e agosto, os indivíduos se alimentam mais, com o intuito de armazenar energia para migração (GODOY, 1975). BARBIERI et al. (2000a) em trabalho relacionando a relação peso-comprimento mostraram resultados semelhantes ao presente trabalho, com peso maior durante a seca.

Foram encontradas no presente trabalho fêmeas maiores e mais pesadas que os machos em $P$. maculatus, resultado também encontrado em outros trabalhos realizados em outros rios com a mesma espécie (SANTOS et al., 2007; Albuquerque et al., 2008; RAmos et al., 2011). O peso e comprimento médios de $P$. maculatus em setembro apresentaram os maiores valores, o que pode estar relacionado ao comportamento dessa espécie, que migra no verão, sendo então encontrados neste período indivíduos adultos (RAmos et al. , 2011), explicando o fato de ter sido um mês em que estes parâmetros foram maiores. Setembro não foi coletado nenhum macho e as fêmeas que se apresentaram no estádio de em maturação e maduro.

Pimelodus maculatus mais coletados foram os imaturos ( $(=81$ ) e em maturação ( $I I=$ 41). No estádio maduro (III=8), foram registradas apenas fêmeas, e no estádio esgotado (IV) não houve nenhum espécime coletado durante o ano. Os estádios imaturos e em maturação foram encontrados durante a maioria dos meses de coleta, exceto no mês de julho que não foi encontrado nenhum indivíduo desta espécie. Nos meses de setembro e novembro não foram coletados machos em nenhum estádio de desenvolvimento. LIMA-JúNIOR \& GOITEIN (2006) observaram que no rio Piracicaba as fêmeas estavam em maturação e no verão encontraram fêmeas maduras e esgotadas, diferindo do presente trabalho, em que na primavera foram encontradas fêmeas nos três estádios de maturação e apenas machos em maturação. No verão foram encontrados indivíduos jovens, em maturação e maduros (apenas fêmeas no último estádio), representando uma diferença em comparação com os resultados dos autores supracitados.

O fator de condição encontrado no presente trabalho mostrou que houve diferença significativa para o parâmetro $b$ para as duas estações do ano em $P$. lineatus, mas apenas a estação cheia apresentou alometria positiva, com incremento de peso maior que de comprimento, mostrando que houve investimento no aumento de massa para o período de migração. Durante a estação seca observou-se que em $P$. maculatus a relação peso-comprimento foi negativa e positiva para a estação cheia, nesta estação foram encontrados indivíduos nos três estádios de maturação (jovens, em maturação e maduros) e na estação seca apenas indivíduos jovens e em maturação. Segundo LIMA-JUNIOR \& GOITEIN (2006) o menor valor para o fator de 
condição encontrado para mandi foi no outono, possivelmente devido ao gasto energético durante a primavera e verão, período que ocorreu a reprodução no rio Piracicaba. No presente trabalho o peso baixo no período de seca está de acordo com o encontrado em trabalhos realizados com a mesma espécie (LIMA-JUNIOR \& GoITEIN, 2006; RAMOs et al., 2011).

A parasitofauna de P. maculatus no rio Mogi Guaçu apresenta semelhanças com a de outros rios estudados (BACHMANN et al., 2007; SANtos et al., 2007; Albuquerque et al., 2008; VENÂNCIO et al., 2010). No presente trabalho 99,2\% dos P. maculatus estavam parasitados por pelo menos uma espécie de parasito, sendo que os mais prevalentes foram os monogenéticos que ocorreram em quase todos os indivíduos coletados. Os parâmetros ecológicos encontrados foram próximos aos encontrados em outros trabalhos como de BACHMANN et al. (2007), no rio Itajaí-Açu que observaram para monogenéticos alta prevalência $(96,3 \%)$, enquanto encontramos uma prevalência de $98,0 \%$ de monogenéticos nas brânquias. SANTOS et al. (2007) encontraram para o rio Guandu uma prevalência de 95,0\% de monogenéticos. Os monogenéticos das cavidades nasais também foram muito prevalentes $(83,08 \%)$. Estes dados mostram que por terem um ciclo direto no hospedeiro, estes parasitos oferecem maior capacidade de infestação.

Outras espécies encontradas neste estudo e que foram semelhantes aos de outros rios foram os Nematoda, C. pinnai pinnai e $P$. (S.) pimelodus, que neste trabalho a prevalência foi de $9,23 \%$ e $27,7 \%$ respectivamente, enquanto SANTOS et al. (2007), encontraram $85 \%$ e $1,66 \%$ respectivamente. Apesar da semelhança de espécies de parasitos, o encontro de um número maior de outras espécies de parasitos para o rio Guandu demonstra uma maior diversidade de parasitos quando comparado às espécies coletadas no presente trabalho.

BACHMANN et al. (2007) encontraram além dos monogenéticos, $P$. (S.) pimelodus com prevalência de $51,2 \%$, também maior que no presente trabalho, mas estes autores não encontraram grande diversidade parasitária. Albuquerque et al. (2008) no rio Guandu, encontraram apenas dois grupos taxonômicos, Nematoda (C. pinnai) e Eucestoda (Nomimoscolex sp.), em $73,42 \%$ dos peixes infectados por pelo menos uma das duas espécies de parasitos.

KoHN et al. (1985) encontraram para coletas realizadas no rio Mogi Guaçu 6 P. maculatus e todos infectados, por pelo menos, uma espécie de parasito, sendo estes 3 monogenoides (Dactylogyroidea sp.), 3 nematoides (C. pinnai) e 3 cestoides (Larva). Os baixos índices ecológicos encontrados são um fator comum neste rio, apresentando uma variação nas espécies de parasitos ao longo de cada ano.

$\mathrm{Na}$ bacia hidrográfica do rio São Francisco foi realizado estudos por BRASIL-SATO (2003), BRAsIL-SATO \& SANTOS (2003), BRASIL- 
SATO \& PAVANelLi (2004) e foi encontrada certa semelhança em relação a helmintofauna entre famílias de peixes nos rios Mogi-Guaçu e Paraná (sub-bacia hidrográfica do rio Paraná) indicando uma ampla distribuição das espécies de parasitos.

\section{AgRADECIMENTOS}

Agradecemos a Coordenação de Aperfeiçoamento de Pessoal de Nível Superior (CAPES) pela concessão da bolsa de doutorado para pesquisa, ao CEPTA, pelo apoio logístico.

\section{REFERÊNCIAS BIBLIOGRÁFICAS}

Aguiar, J. C., Ceccarelli, P. S., \& Luque, J. L. 2011. Two new species of Pavanelliella (Monogenea, Dactylogyridae) parasitic on pimelodid fishes from Mogi Guaçu river, Southeastern Brazil, and notes on the morphology of $P$. pavanellii. Neotropical Helminthology 5(2): 213-224.

Albuquerque, M. C.; Santos, M. D.; Monteiro, C. M.; Martins, A. N. \& EderLI, N. B. 2008. Helmintos endoparasitos de Pimelodus maculatus Lacépède, 1803 (Siluriformes, Pimelodidae) de duas localidades (lagoa e calha) do Rio Guandu, Estado do Rio de Janeiro, Brasil. Revista Brasileira de Parasitologia Veterinária 17: 113-119.

AMAto, J.F.R; W.A. Boeger \& AMATO, S.B. 1991. Protocolos para laboratório Coleta e Processamento de Parasitos de Pescado. Imprensa Universitária. Universidade Federal Rural do Rio de Janeiro. 81pp.

Bachmann, F.; Greinert, J. A.; Bertelli, P. W.;
Da Silva Filho, H. H.; DE LaRa, N. O. T.; GHIRALDELLI, L. \& MARTINS, M. L. 2007. Parasitofauna de Pimelodus maculatus (Osteichthyes: Pimelodidae) do rio ItajaíAçu em Blumenau, Estado de Santa Catarina, Brasil. Acta Scientiarum Biological Sciences 29 (1): 109-114.

Barbieri, G.; Salles, F. A. \& Cestarolli, M. A. 2000a. Influência de fatores abióticos na reprodução do dourado, Salminus maxillosus e do curimbatá, Prochilodus lineatus do rio Mogi Guaçu (Cachoeira de Emas, Pirassununga, SP). Acta Limnologica Brasiliensia 12: 85-91.

Barbieri, G.; Salles, F. A. \& Cestarolli, M. A. 2000b. Análise populacional do curimbatá, Prochilodus lineatus, do rio Mogi Guaçu, Pirassununga/SP (Characiformes, Prochilodontidae). Boletim Instituto de Pesca 26 (2): 137-145.

Barbieri, G.; Salles, F. A.; Cestarolli, M. A. TEIXEIRA-FILHo, \& A. R. 2004. Estratégias reprodutivas do dourado, Salminus maxillosus e do curimbatá, Prochilodus lineatus no rio Mogi Guaçu, estado de São Paulo, com ênfase nos parâmetros matemáticos da dinâmica populacional. Acta Scientiarum Biological Sciences 26: 169-174.

Brasil-SATo, M. C. 2003. Parasitos de peixes da bacia do São Francisco, pp. 149-165. In: H. P. Godinho, A. L. Godınho (eds.). Águas, Peixes e Pescadores do São Francisco das 
Minas Gerais. Belo Horizonte, PUC Minas. 468p.

BRASIL-SATO, M. C; SANTOS, M. D. 2003. Helmintos de Myleus micans (Lütken, 1875) (Characiformes: Serrasalminae) do rio São Francisco, Brasil. Revista Brasileira de Parasitologia Veterinária 12(3): 131-134.

Brasil-Sato, M. C. \& Pavanelli, G. C. 2004. Digenea de Pimelodus maculatus (Osteichthyes, Pimelodidae) das bacias dos rios São Francisco e Paraná, Brasil. Parasitología Latinoamericana 59: 123-131.

Bush, A. O., LAFFERTY, K. D., LOTZ, J. M., \& SHOSTAK, A. W. 1997. Parasitology meets ecology on its own terms: Margolis et al. revisited. Journal of Parasitology: 575-583.

Capeleti, A. R. \& Petrete JR., M. 2006. Migration of the curimbatá Prochilodus lineatus (Valenciennes, 1836) (Pisces, Prochilodontidae) at the waterfall "Cachoeira de Emas" of the Mogi Guaçu river - São Paulo, Brazil. Brazilian Journal of Biology 66(2b): 651-659.

Cepeda, P. B., Ceccarelli, P. S., \& Luque, J. L. 2012. Una nueva especie de Tereancistrum (Monogenea, Dactylogyridae) parásito de Prochilodus lineatus (Valenciennes, 1837) (Characiformes) del Rio Mogi Guaçu, Brasil. Neotropical Helminthology 6(2): 205-211.

EspíndolA, E. L. G.; BRIGANTE, J. \& ELER, M. N. 2003. Avaliação ambiental preliminar do uso e ocupação do solo da bacia hidrográfica do rio Mogi Guaçu, pp.23-53. In: BRIGANTE \& ESPÍNDOLA (eds.). Limnologia fluvial: um estudo no Rio Mogi Guaçu. 278p.

Godoy, M. P. 1975. Peixes do Brasil, subordem Characoidei: bacia do rio Mogí Guassú. Vol. IV. Editora Franciscana, Piracicaba, 489p.

KRITSKY, D.C; VIANNA, R.T. \& BOEGER, W.A. 2007. Neotropical Monogenoidea. 50. Oviparous gyrodactylids from loricariid and pimelodid catfishes in Brazil, with the proposal of Phanerothecioides n. g., Onychogyrodactylus n. g. and Aglaiogyrodactylus n. g. (Polyonchoinea: Gyrodactylidea). Systematic Parasitology 66: 1-34

Kohn, A., Fernandes, B. M. M., Macedo, B., \& ABRAMSON, B. 1985. Helminths parasites of freshwater fishes from Pirassununga, SP, Brazil. Memórias Instituto Oswaldo Cruz 80(3): 327-336.

KOhn A. \& Fernandes, B. M. М. 1987. Estudo comparativo dos helmintos parasitos de peixes do rio Mogi Guassu, coletados nas excursões realizadas entre 1927 e 1985. Memórias Instituto Oswaldo Cruz 82: 483-500.

LIMA-JUNIOR, S. E. \& GOITEIN, R. 2006. Fator de condição e ciclo gonadal de Pimelodus maculatus (Osteichthyes, Pimelodidade) no rio Piracicaba (SP, Brasil). Boletim 
Instituto Pesca 32(1): 87-94.

Lizama, M. A. P.; TAKemoto, R. M. \& PAVANelli, G. C. 2005. Influence of host sex and age on infracommunities of metazoan parasites of Prochilodus lineatus (Valenciennes, 1836) (Prochilodontidae) of the Upper Paraná River floodplain, Brazil. Parasite 12: 299304.

MESQUitA, R. L. B. 2011. Helmintos endoparasitas de 16 espécies de peixes characiformes e siluriformes do rio Mogi Guaçu, Pirassununga, estado de São Paulo, Brasil. Dissertação de Mestrado. Universidade Federal Rural do Rio de Janeiro, 76p.

Ramos, I. P.; VidotTo-Magnoni, A. P.; BRANDÃo, H.; David, G. S. \& Carvalho, E. D. 2011. Feeding, reproduction and population structure of Pimelodus maculatus Lacépéde, 1803 (Teleostei, Siluriformes, Pimelodiae) from Paraná basin: a review. Boletim Associação Brasileira Limnologia 39: 1-15.

SAntos, M. D.; Lemos-PitA, S. R. L. C. \& BRASILSATO, M. C. 2007. Metazon parasite fauna of Pimelodus maculatus Lacépède, 1803 (Siluriformes, Pimelodidade) from the Guandu river, Rio de Janeiro state, Brazil. Acta Scientiarum Biological Sciences 29(1): 101-107.

Takemoto, R. M.; Pavanelli, G. C.; lizama, M. A. P.; Lacerda, A. C. F.; Yamada, F. H.;
MoreirA, L. H. A.; CeschinI, T. L. \& Bellay, S. 2009. Diversity of parasites of fish from the Upper Paraná River floopplain, Brazil. Brazilian Journal of Biology 69(2): 691705.

VAzzoler, A. E. A. D. M. 1981. Manual de métodos para estudos biológicos de populações de peixes: reprodução e crescimento. Brasília, CNPq, 108p.

VenÂncio, A. C. P.; Aguiar, G. R. D.; LOPES, P. D. S. \& ALVES, D. R. 2010. Metazoan parasites of mandi-amarelo Pimelodus maculatus and of jundiá Rhamdia quelen (Osteichthyes: Siluriformes) of Paraíba do Sul river, Volta Redonda, Rio de Janeiro. Revista Brasileira Parasitologia Veterinária 19(3): 157-163.

Vicentin, W.; RochA, A. S.; RONDON, P. L.; COSTA, F. E. S. \& SúAREZ, Y. R. 2012. Parâmetros populacionais, período reprodutivo e crescimento de Prochilodus lineatus (Characiformes, Prochilodontidae) na cabeceira do rio Miranda, alto rio Paraguai. Oecologia Australis 16: 891-904.

Recebido: $18 / 10 / 2016$

Revisado: 20/02/2017

Aceito: $24 / 03 / 2017$ 
106. REIS et al.

Revista Brasileira de Zoociências 18(2): 91-106. 2017 